\title{
Green Sonochemical Synthesis of Silver Nanoparticles Using Adansonia Digitata Leaves Extract and Evaluation of Their Antibacterial Potential
}

\author{
Wilson Njue, Jackson Kilonzo Kithokoi, Jane Mburu, Henry Mwangi, Sauda Swaleh.
}

\section{ABSTRACT}

\begin{abstract}
Metal nanoparticles in the field of nanotechnology are of great interest to modern scientific research due to their size effects, medical uses and, catalytic, electronic and optical properties. Green synthesis of metal nanoparticles is a feasible alternative to chemical methods as it is environmentally friendly and cost effective. In continuation with our research on green synthesis of silver nanoparticles using Kenyan medicinal plants, we here report the synthesis of novel silver nanoparticles (AgNPs) on ultrasonic bath using Adansonia digitata leaves extracts and analysis of their antibacterial activity. The nanoparticles were characterized by UV-Vis, High Resolution Transmission Electron Microscopy (HRTEM), FTIR spectroscopy and Energy Dispersive X-ray (EDX). EDX analysis affirmed the nanoparticles were pure silver. Crystalline nature of the nanoparticles was confirmed by bright circular spots in the Selected Area Electron Diffraction (SAED) in HRTEM image. The AgNPs were spherical with an average size $13 \mathrm{~nm}$. FTIR analysis showed strong $-\mathrm{C}=\mathrm{C}$ - and $-\mathrm{OH}$ stretching bands due to compounds capping the nanoparticles. The synthesized AgNPs showed high inhibition zones of $17.1 \pm 0.130 \mathrm{~mm}$ towards Gram-negative bacteria $E$. coli and $12.9 \pm 0.082 \mathrm{~mm}$ towards Gram positive bacteria $S$. aureus. The aqueous $A$. digitata extract had no effect on growth inhibition of test bacteria. The study showed that the silver nanoparticles synthesized from the plant's leaves extract had antibacterial activity against both Gram negative and positive pathogenic bacteria. The nanoparticles can be utilized towards developing novel drugs useful in combating pathogens.
\end{abstract}

Keywords: A. digitata, EDX, HRTEM, Minimum Inhibitory Concentration, ultrasonic.bath
Published Online: May 22, 2020

ISSN: $2684-4478$

DOI : $10.24018 /$ ejchem.2020.1.2.5

Wilson Njue*

Kenyatta University, Kenya.

(e-mail: wilsonnjue@yahoo.com)

Jackson Kilonzo Kithokoi

Kenyatta University, Kenya.

(e-mail: jkilonzo22@gmail.com)

Jane Mburu

Kenyatta University, Kenya.

(e-mail: jane.mburu@gmail.com)

Henry Mwangi

Kenyatta University, Kenya.

(e-mail: henrimwangi@gmail.com)

Sauda Swaleh

Kenyatta University, Kenya.

(e-mail: swaleh.sauda@ku.ac.ke)

\section{INTRODUCTION}

Nanotechnology is an important field of modern research dealing with synthesis, strategy and manipulation of particles of size ranging from approximately 1 to $100 \mathrm{~nm}$. It has the potential to create new materials and certainly provide answers to world's problems related to agriculture, nutrition, health, water and energy [1]. Metal nanoparticles with controlled shape and size form basis for advanced functional materials for electronic, medical sensor and optical devices. Generally, metal nanoparticles are prepared by physical and chemical methods which are costly and potentially harmful to the environment. The chemical methods involve use of chemicals like Sodium borohydride, Sodium Citrate, hydrazine and Ascorbate, some of which are toxic. $\mathrm{NaBH}_{4}$ can be a source of caustic salts and flammable gases, which contribute to various biological risks [2]. Green synthesis of nanoparticles has received a lot of attention due to need to develop environmentally friendly methods in materials processing. The green process is rapid, economical, nonpathogenic and ecofriendly [3]. Equally, biological synthesis of silver nanoparticles (AgNPs) is on limelight because of their diverse range of applications in bioengineering, food packaging [4], medical industry [5], cosmetics [6], catalysis, electrochemistry and water treatment [7].

The green synthesis technique for assembly of AgNPs involve use of plant extracts, fungi, bacteria, molds and biodegradable polymers [8], [9]. Plant extracts are novel, greener, cost-effective and friendly to environment hence can be used in the synthesis of AgNPs useful for medical applications [10]. Biomolecules like proteins, alkaloids, flavonoids, phenolic compounds, vitamins like vitamin $\mathrm{C}$, polysaccharides and terpernoids in the plants have been implicated as reducing, capping and stabilizing agents in the assembly of AgNPs [11]. Plants synthesized nanoparticles are more stable and are produced faster than those synthesized by microorganism [12].

Different plant extracts produce AgNPs of different sizes 
and shapes, and in certain cases they produce mixed shapes [13]. AgNPs have been synthesized via sonochemical methods by applying ultrasound radiation $(20 \mathrm{KHz}-10 \mathrm{MHz})$ or use of ultrasonic bath (sonicator) where nanoparticles of different sizes were formed [14]. The physical phenomenon in sonochemistry involves cavitation and nebulization. AgNPs of size range $4-21 \mathrm{~nm}$ have been synthesized via sonochemical method using $k$-carageenan sea weed extract as reducing and stabilizing agent [15]. Also, AgNPs of size $100 \mathrm{~nm}$ have been synthesized using citrate by sonication [16]. In addition, Prunus Africana and Bridelia micrantha plant extracts have been used in synthesis of AgNPs of sizes, 23 and $16 \mathrm{~nm}$ respectively by sonochemical method. The nanoparticles were spherical and evenly distributed [17], [18].

Plant selected for this study was Adansonia digitita (Boabab) also called by Kenyan local names, kiamba (Kamba), mbuyu (Swahili). It is found in the lower Eastern and Coastal regions of Kenya. A. digitata leaves contain vitamin $\mathrm{C}$, carbohydrates, polyphenols carbohydrates and show high antioxidant capacity [19]. The young leaves were found to be rich in calcium, iron, zinc and vitamin $C$ [20]. Ultrasonic bath facilitated the synthesis of AgNPs in this research.

\section{MATERIALS AND METHODS}

\section{A. Sample Collection}

A. digitata leaves (Fig.1) were collected from Makueni County, Kenya. The plant specimen was identified by taxonomist from Department of Plant Sciences and voucher specimen deposited at the herbarium in Kenyatta University.

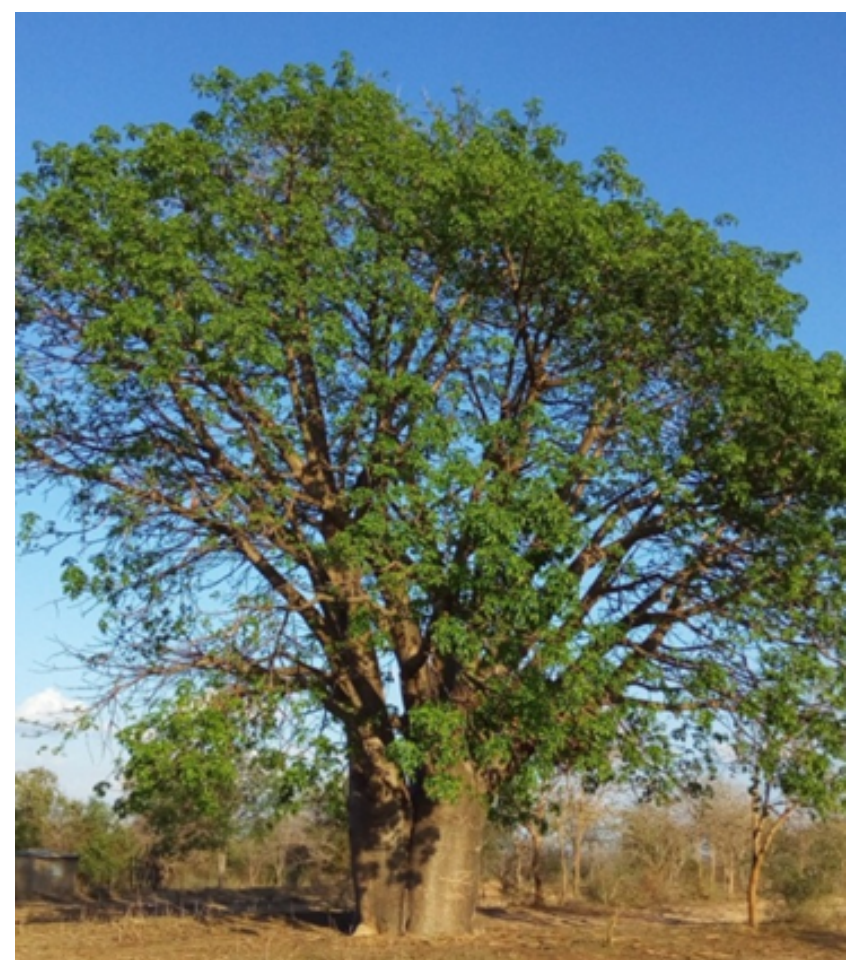

Fig. 1. Adansonia digitata (boabab)

\section{B. Chemicals, Apparatus and Reagents}

All the chemicals used in this study were of analytical grade. Potassium bromide, Mueller Hinton, Nutrient agar, silver nitrate $(99.8 \%)$ and nitric acid were obtained from Sigma- Aldrich (Germany). All solutions were freshly prepared using double distilled water. The glassware used was cleaned in fresh solution of nitric acid, washed thoroughly with tap water, rinsed with distilled water and dried in the oven before use.

\section{Sample Preparation}

The plant leaves were cleaned using water to remove impurities, chopped and air dried for two weeks at room temperature. A grinder (Retsch 200, Germany), was used to pulverize the leaves into fine powder. The plant extract was prepared by mixing $10 \mathrm{~g}$ of the dried powder with $100 \mathrm{~mL}$ of distilled water then heated for 20 minutes at $60{ }^{\circ} \mathrm{C}$. The extract was filtered using Whatman filter paper No. 1 to obtain a clear filtrate. The filtrate was then centrifuged for 10 minutes to remove the fine plant particles. The extract was stored at $-4{ }^{\circ} \mathrm{C}$ for further use.

\section{Ultrasonic synthesis of AgNPs}

The synthesis was done using ultrasonic bath (WUC$\mathrm{AO} 3 \mathrm{H})$ as agitator as described by [21] with some modification. $90 \mathrm{ml}$ of $0.001 \mathrm{M}$ silver nitrate solution was mixed with $10 \mathrm{ml}$ of the plant extract in a conical flask. The flask was immersed in the ultrasonic bath and the formation of AgNPs monitored through colour change of the mixture.

\section{E. UV-visible spectroscopy}

UV-Vis spectrophotometer (Specord 200 analytik Jena) was used to monitor the formation of AgNPs. The scanning was done at regular intervals to check the intensity of surface plasmon resonance band in the range of $400-450$ $\mathrm{nm}[22]$. Water was used as blank.

\section{F. Fourier Transform Infra-Red Spectroscopy (FTIR) Analysis}

FTIR spectrometer (Shimadzu IRT Technai racer - 100) was used to determine the functional groups of compounds responsible for capping and stabilizing the AgNPs. The sample solution containing the nanoparticles was centrifuged at $5000 \mathrm{rpm}$ for 20 minutes, filtered then evaporated. The solid material was mixed with Potassium bromide then pelleted with a mini- press. The pellets were used for analysis.

G. High Resolution Transmission Electron 


\section{Microscope (HRTEM) analysis}

HRTEM (FEI Tecnai F20) coupled with Energy Dispersive X-ray (EDX) was used to determine the size, shape, morphology and nature of nanoparticles. The samples for analysis were prepared by drop coating the AgNPs solution on to carbon - coated copper TEM grids [23].

\section{H. Antibacterial activity}

The antibacterial activity of AgNPs was done using the paper disc diffusion technique. The test bacterial strains were sub cultured for 24 hours. The concentration of the bacteria was determined by comparing its turbidity with McFarland solution. The inoculum was swabbed on the nutrient agar in sterile petri dishes. Paper discs $(6 \mathrm{~mm})$ impregnated with AgNPs were placed on the same petri dishes then incubated for 24 hours. Zones of inhibition were measured after 24 hours. The magnitude of antimicrobial effect against, Escherichia coli and Staphylococcus aureus was determined based on the inhibition zone [11]. The AgNPs exhibiting activity had the minimum inhibitory concentration (MIC) determined.

\section{RESULTS AND DISCUSSIONS}

\section{A. Synthesis of AgNPs}

The colour of solution mixture of the A. digitata leaves extract and $\mathrm{AgNO}_{3}$ (aq) changed from pale yellow to light brown, red brown then finally dark brown in 20 minutes (Fig. 2). There are no previous records showing studies done on synthesis of AgNPs using A. digitata leaves under ultrasonic bath.

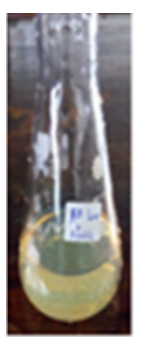

(i)

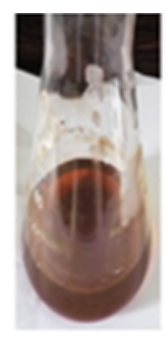

(ii)

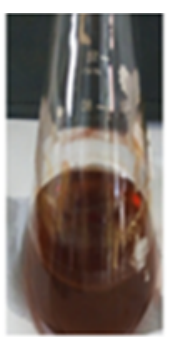

(iii)

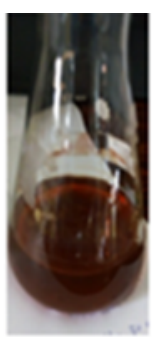

(iv)



(v)
Fig. 2. A digitata leaves extract and $\mathrm{AgNO}_{3}$ solution at (i) 0 , (ii) 5 (iii) 10 (iv) 15 (v) 20 minutes of sonication

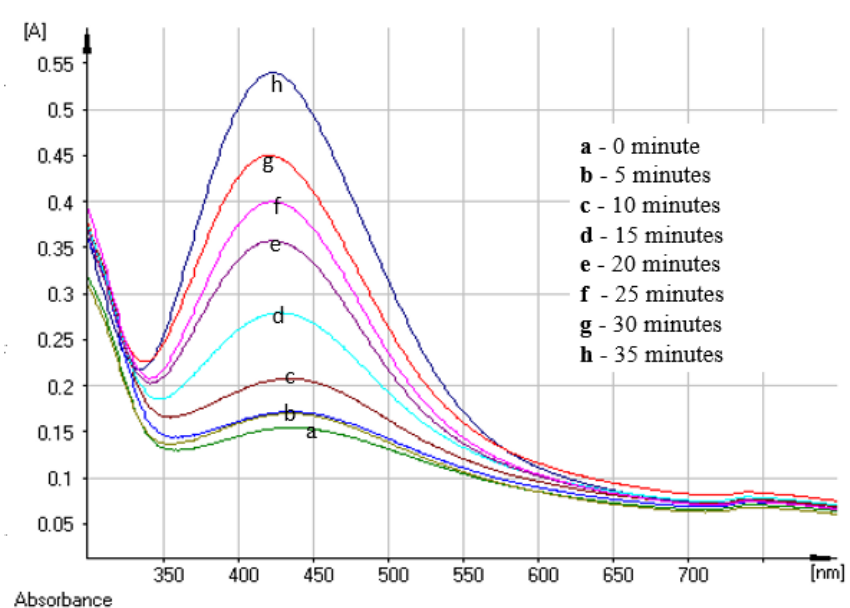

ig. 3. UV-Vis spectra of AgNPs prepared using A. digitata plant extract as a function of time.

Colour change of the solution to brown was an indication of the formation of AgNPs as observed in previous studies on use of plants to synthesize AgNPs [24]. The formation of silver nanoparticles was further characterized using UV-Vis spectroscopy. The surface plasmon resonance (SPR) bands (Fig. 3), characteristic of AgNPs were detected between 425 $-448 \mathrm{~nm}$. The absorbance peaks were observed at different times after the reaction started. The SPR bands are influenced by size, shape, morphology, composition and dielectric environment of nanoparticles [25].

The peaks at 5 and 10 minutes were broad indicating that the AgNPs formed were polydispersed. Broadening of peaks in the UV-Vis spectra was also observed during the study of Optical properties and ultrafast dynamics of metallic nanocrystals [26]. The peaks narrowed after 15 minutes an indication of reduction in size of nanoparticles. The absorbance intensity increased steadily up to 35 minutes, indicating increase in the concentration of AgNPs. Increase in absorbance with time was also noted in the study on biosynthesis of AgNPs using Diopyros kaki leaves where absorbance of absorption peaks at $\lambda_{\max } 430 \mathrm{~nm}$ increased in intensity up to 480 minutes [9].

The blue shift in wavelength from $448 \mathrm{~nm}$ to $425 \mathrm{~nm}$ indicated decrease in size of the synthesized AgNPs. Similar observation on decrease in size of AgNPs under sonication was also made by Khan and others [27] in their study on applications of plant terpenoids in the synthesis of colloidal silver nanoparticles. The decrease in size of the AgNPs during synthesis can be attributed to ultrasound cavitation phenomenon during sonication [28] and also the decrease in concentration of the reactants with time [29].

\section{B. High Resolution Transmission Electron Microscope (HRTEM) Analysis}

HRTEM images of synthesized silver nanoparticles are presented in Figure 4 (images A to E).The AgNPs were spherical and evenly distributed. The images showed that, there was no agglomeration of the silver nanoparticles. The biomolecules in $A$. digitata leaves extract reduced $\mathrm{Ag}^{+}$ions to stable $\mathrm{Ag}^{0}$ atoms without clustering. The surface of the spherical nanoparticles were non-uniform as indicated in Figure 4, image F. 


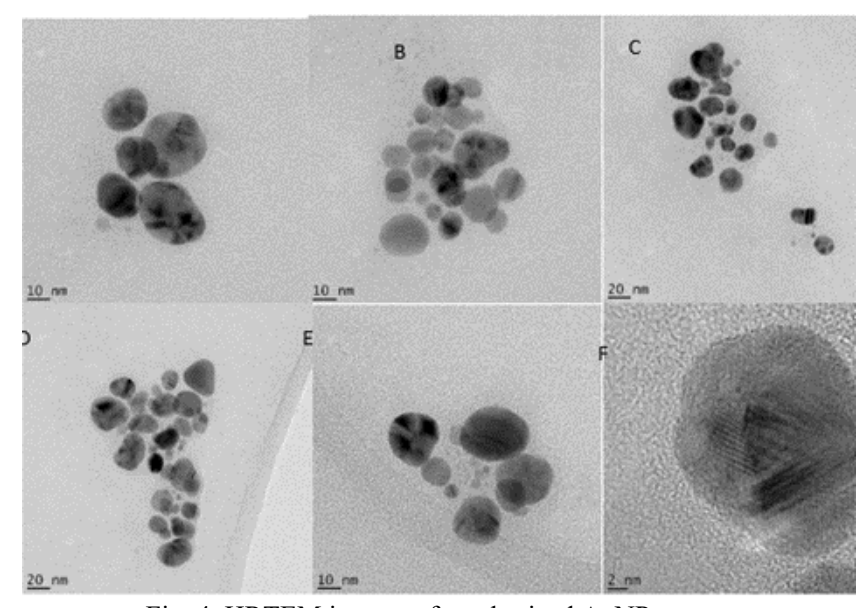

Fig. 4. HRTEM images of synthesized AgNPs.

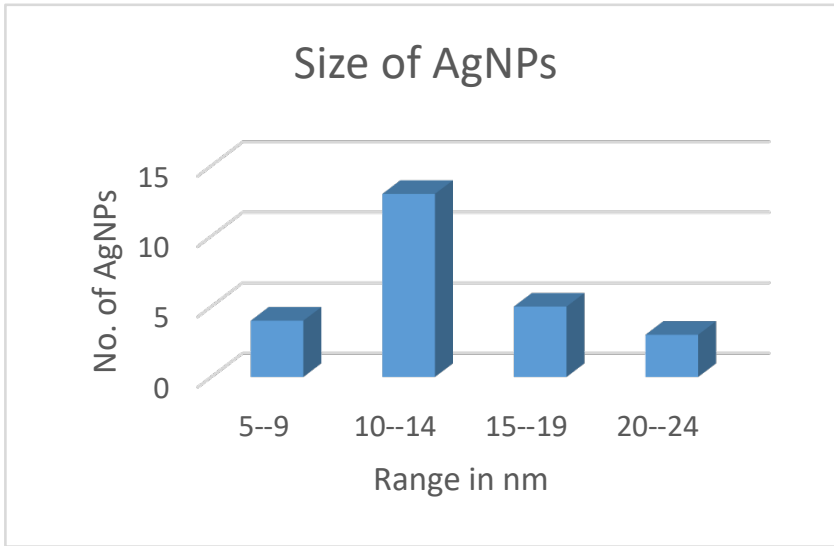

Fig. 5. Size distribution of AgNPs

The sizes of the synthesized AgNPs from HRTEM analysis were in the range of 5 to $24 \mathrm{~nm}$ (Fig. 5). The results showed that the synthesized nanoparticles were monodispersed which concurs with UV-Vis analysis. The average size of the synthesized AgNPs was $13 \mathrm{~nm}$. The AgNPs synthesized in this study were smaller in size (5 to $24 \mathrm{~nm}$ ) than those obtained ( 3 to $57 \mathrm{~nm}$ ) in the synthesis of AgNPs using Kigeria africana plant extract [31].

\section{Selected Area Electron Diffraction (SAED) Analysis}

The Selected Area Electron Diffraction (SAED) micrograph (Fig. 6) depicted discrete shiny circular spots, confirming the crystallinity of AgNPs. [31] .

\section{Energy Dispersive X-ray (EDX) Analysis}

Energy Dispersive X-ray (EDX) spectroscopy was used to show the elemental composition of the sample. Figure.7 shows an EDX spectrum with peaks at around $3.0 \mathrm{keV}$ which were due to elemental silver. The peaks in EDX are in agreement with observation made on synthesis of AgNPs using Adansonia digitata [32].

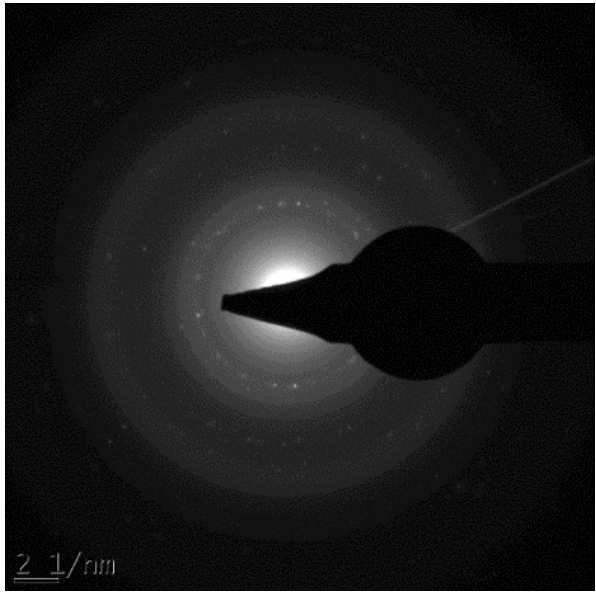

Fig. 6 SAED micrograph of AgNPs.

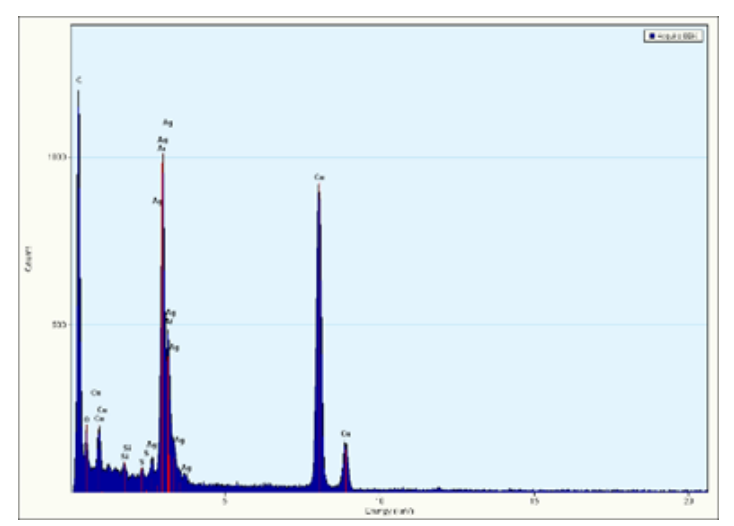

Fig. 7. EDX spectrum of AgNPs .

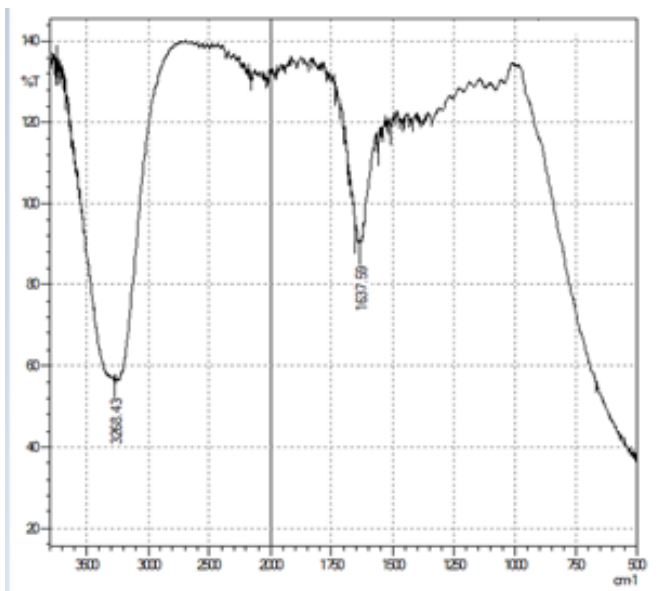

Fig. 8. FTIR spectrum of AgNPs

E. Fourier Transform Infra-Red (FTIR) analysis of AgNPS

FTIR spectrum for AgNPs (Fig. 8) showed strong broad peak at $3268 \mathrm{~cm}^{-1}$ due to O-H stretching and at $1637 \mathrm{~cm}^{-1}$ for $-\mathrm{C}=\mathrm{C}$ - stretching. The functional groups could be due to phenolic compounds capping and stabilizing the AgNPs. The same functional groups were observed on AgNPs synthesized using $A$. digitata leaves [32]. 


\section{F. Antibacterial activity of AgNPS}

The synthesized nanoparticles inhibited the growth of both Gram-positive and Gram-negative bacteria. AgNPs exhibited inhibition zone of $17.1 \pm 0.130 \mathrm{~mm}$ towards Gramnegative bacteria ( $E$. coli) against the positive control (Ciprofloxacin) with inhibition zone of $33.4 \pm 0.443$ and inhibition zone of $12.9 \pm 0.082 \mathrm{~mm}$ towards Gram-positive bacteria, $S$. aureus against an inhibition zone of $22.5 \pm 0.988$ of positive control (Vancomycin). The aqueous plant extract had no effect on inhibition of growth of bacteria. Zones of inhibition give in Table 1 and figure 9. 
TABLE I: INHIBITION ZONES OF AGNPS AGAINST E. COLI AND S. AUREUS

\begin{tabular}{ccc}
\hline \multicolumn{1}{c}{ Sample } & \multicolumn{2}{c}{ Zones of inhibition in mm } \\
& $E$. coli & S. aureus \\
\hline AgNPs & $17.1 \pm 0.13$ & $12.9 \pm 0.08$ \\
Ciprofloxacin & $33.4 \pm 0.44$ & N/A \\
Vancomycin & N/A & $22.5 \pm 0.98$ \\
Negative control (water) & 6 & 6 \\
Aqueous leaves extract & 6 & 6 \\
\hline
\end{tabular}

NB: N/A- not applicable



Fig. 9. Zones of inhibition of AgNPs against (A)- S. aureus and (B)- E.coli.

Studies done by [32] showed AgNPs from A. digitata leaves of size 5 to $10 \mathrm{~nm}$ had antibacterial activity of $24.1 \pm 0.60 \mathrm{~mm}$ on $E$. coli. The higher antibacterial activity in his study could have been due to the smaller size of AgNPs. AgNPs can penetrate through the bacteria cell membrane and interact with compounds containing sulphur and phosphorous like proteins and DNA thus making the bacteria to lose viability and eventually lead to death [33]. The results of bacterial inhibition by synthesized silver nanoparticles showed that; $S$. aureus was susceptible to the AgNPs while $E$. coli was intermediate.

The minimum Inhibitory concentration (MIC) of AgNPs was determined by doing replicate tests. For $E$. coli it was $0.125 \mathrm{mM}$ while for $S$. aureus it was $0.25 \mathrm{mM}$. The clear hollows in Fig. 9 shows some zones of inhibition of different concentrations of AgNPs on S. aureus: 1-1mM, 2$0.75 \mathrm{mM}, 3-0.5 \mathrm{mM} 4-0.25 \mathrm{mM}$ and E- the plant extract.

\section{CONCLUSION}

Green sonochemical synthesis of AgNPs was achieved by use of $A$. digitata leaves extract as reducing, capping, and stabilizing agent. The formation AgNPs was confirmed by the UV-VIS absorption spectroscopy which showed surface plasmon resonance (SPR) band characteristic of AgNPs in the range of 425- $448 \mathrm{~nm}$. FTIR spectrum of the nanoparticles showed strong $-\mathrm{OH}$ and $-\mathrm{C}=\mathrm{C}$ - stretching, due to functional groups of biomolecules capping the AgNPs. HRTEM images showed the AgNPs were monodispersed, spherical and had an average size of $13 \mathrm{~nm}$. SAED analysis showed the synthesized AgNPs were crystalline. This study showed that AgNPs can be synthesized using A. digitata leave extracts under sonication and the two strains of bacteria are susceptible to the AgNPs. The synthesized AgNPs have the potential of being used in development of new drugs to fight pathogenic bacteria.

\section{ACKNOWLEDGMENT}

The authors wishes to acknowledge Kenyatta University, Vice Chancellor's research grant and Department of Chemistry, Kenyatta University for support of this research. In addition the authors acknowledge Prof. Martin Onani, University of the Western Cape for facilitating the EDX/HRTEM analysis.

\section{REFERENCES}

[1] Bonnia, N., Kamaruddin, M., Nawawi, M., Ratim, S., Azlina, H. and Ali, E. Green biosynthesis of silver nanoparticles using 'Polygonum hydropiper'and study its catalytic degradation of methylene blue. Procedia Chemistry, 19 (2016) 594-602.

[2] Arya, V. (2010). Living systems: eco-friendly nanofactories. Digest Journal of Nanomaterials and Biostructures, 5 (1) 9-21.

[3] Mukherjee, P., Roy, M., Mandal, B., Dey, G., Mukherjee, P., Ghatak, J., Tyagi, A. and Kale, S. Green synthesis of highly stabilized nanocrystalline silver particles by a non-pathogenic and agriculturally important fungus T. asperellum. Nanotechnology, 19 (7) (2008) 075103.

[4] Duncan, T. V. Applications of nanotechnology in food packaging and food safety: barrier materials, antimicrobials and sensors. Journal of colloid and interface science, 363 (1) (2011) 1-24.

[5] Prabhu, S. and Poulose, E. K. Silver nanoparticles: mechanism of antimicrobial action, synthesis, medical applications, and toxicity effects. International Nano Letters, 2 (1) (2012) 32.

[6] Keller, A. A., McFerran, S., Lazareva, A. and Suh, S. Global life cycle releases of engineered nanomaterials. Journal of Nanoparticle Research, 15 (6) (2013) 1692

[7] Bystrzejewska-Piotrowska, G., Golimowski, J. and Urban, P. L. Nanoparticles: their potential toxicity, waste and environmental management. Waste management, 29 (9) (2009) 2587-2595.

[8] Song, J. Y. and Kim, B. S. Rapid biological synthesis of silver nanoparticles using plant leaf extracts. Bioprocess and biosystems engineering, 32 (1) (2009a) 79-84.

[9] Song, J. Y. and Kim, B. S. Rapid biological synthesis of silver nanoparticles using plant leaf extracts. Bioprocess and biosystems engineering, 32 (1) (2009b).79.

[10] Nagababu, P. and RAO, U. costğeffective green synthesis and characterization of silver nanoparticles from avicennia alba blume leaves and their antibacterial activity. asian $\mathrm{j}$ pharm clin res, 9 (1) (2016) 301-303

[11] Gebru, H., Taddesse, A., Kaushal, J. and Yadav, O. Green synthesis of silver nanoparticles and their antibacterial activity. Journal of Surface Science and Technology, 29 (1-2) (2013) 47-66.

[12] Firdhouse, M. J. and Lalitha, P. Green synthesis of silver nanoparticles using the aqueous extract of Portulaca oleracea (L.). Asian Journal of Pharmaceutical and Clinical Research, 6 (1) (2012) 92-94.

[13] Iravani, S. Green synthesis of metal nanoparticles using plants. Green Chemistry, 13 (10) (2011) 2638-2650.

[14] Esmaeili-Zare, M., Salavati-Niasari, M. and Sobhani, A. Simple sonochemical synthesis and characterization of $\mathrm{HgSe}$ nanoparticles. Ultrasonics sonochemistry, 19 (5) (2012) 1079-1086.

[15] Elsupikhe, R. F., Shameli, K., Ahmad, M. B., Ibrahim, N. A. and Zainudin, N. Green sonochemical synthesis of silver nanoparticles at varying concentrations of $\kappa$-carrageenan. Nanoscale research letters, 10 (1) (2015) 302.

[16] Skiba, M., Pivovarov, A., Makarova, A. and Vorobyova, V. Plasmachemical Synthesis of Silver Nanoparticles in the Presence of Citrate. Chemistry Journal of Moldova: General, 13 (1) (2018) 7-14.

[17] Kilonzo, J.K., Ochoo, L., Maingi, J.M. , Swaleh, S. and Njue W. M. Ultrasonic Synthesis of Silver Nanoparticle mediated by Prunus africana plant extract and their Antibacterial activity. International journal of Nano and Material sciences, 7 (1) (2018a) 43 - 56.

[18] Kilonzo, J.K., Ochoo, L., Maingi, J.M. , Swaleh, S. and Njue W. M. Sonochemical Biosynthesis of Silver Nanoparticle using Bridelia micrantha and evaluation of their Antibacterial activity. European International journal of Science and Technology. Vol.7 No. 9 (2018b) $1-13$.

[19] Ibrahima, C., Didier, M., Max, R., Pascal, D., Benjamin, Y. and Renaud, B. Biochemical and nutritional properties of baobab pulp from endemic species of Madagascar and the African mainland. African Journal of Agricultural Research, 8 (47) (2013) 6046-6054. 
[20] Gebauer, J., El-Siddig, K. and Ebert, G. Baobab (Adansonia digitata L.): a Review on a Multipurpose Tree with Promising Future in the Sudan/Baobab (Adansonia digitata L.): Ein Überblick über eine vielseitig verwendbare Baumart mit guten Zukunftsaussichten für den Sudan. Gartenbauwissenschaft, (2002) 155-160.

[21] Mason, T. J. Ultrasound in synthetic organic chemistry. Chemical Society Reviews, 26 (6) (1997) 443-451.

[22] Rashid, M. U., Bhuiyan, M. K. H. and Quayum, M. E. Synthesis of silver nano particles (Ag-NPs) and their uses for quantitative analysis of vitamin $\mathrm{C}$ tablets. Dhaka University Journal of Pharmaceutical Sciences, 12 (1) (2013) 29-33.

[23] Umashankari, J., Inbakandan, D., Ajithkumar, T. T. and Balasubramanian, T. Mangrove plant, Rhizophora mucronata (Lamk, 1804) mediated one pot green synthesis of silver nanoparticles and its antibacterial activity against aquatic pathogens. Aquatic biosystems, 8 (1) (2012) 11.

[24] Kumar, D. A. Rapid and green synthesis of silver nanoparticles using the leaf extracts of Parthenium hysterophorus: a novel biological approach. Int Res J Pharm, 3 (2) (2012) 169-172.

[25] Shameli, K., Bin Ahmad, M., Jazayeri, S. D., Sedaghat, S., Shabanzadeh, P., Jahangirian, H., Mahdavi, M. and Abdollahi, Y. Synthesis and characterization of polyethylene glycol mediated silver nanoparticles by the green method. International journal of molecular sciences, 13 (6) (2012) 6639-6650

[26] Link, S. and El-Sayed, M. A. Optical properties and ultrafast dynamics of metallic nanocrystals. Annual review of physical chemistry, 54 (1) (2003) 331-366.

[27] Khan, M. A., Khan, T. and Nadhman, A. Applications of plant terpenoids in the synthesis of colloidal silver nanoparticles. Advances in colloid and interface science, 234 (2016) 132-141.

[28] Yin, Y., Li, Z.-Y., Zhong, Z., Gates, B., Xia, Y. and Venkateswaran, S. Synthesis and characterization of stable aqueous dispersions of silver nanoparticles through the Tollens process. Journal of Materials Chemistry, 12 (3) (2002) 522-527.

[29] Prathna, T., Chandrasekaran, N., Raichur, A. M. and Mukherjee, A. Biomimetic synthesis of silver nanoparticles by Citrus limon (lemon) aqueous extract and theoretical prediction of particle size. Colloids and Surfaces B: Biointerfaces, 82 (1) (2011) 152-159.

[30] Rajeshkumar, S. and Veena, P. Biomedical Applications and Characteristics of Graphene Nanoparticles and Graphene-Based Nanocomposites Exploring the Realms of Nature for Nanosynthesis : Springer. (2018) 341-354

[31] Ashishie, P. B., Anyama, C. A., Ayi, A. A., Oseghale, C. O., Adesuji, E. T. and Labulo, A. H. Green synthesis of silver monometallic and copper-silver bimetallic nanoparticles using Kigelia africana fruit extract and evaluation of their antimicrobial activities. International Journal of Physical Sciences, 13 (3) (2018) 24-32.

[32] Kumar, C. M. K., Yugandhar, P. and Savithramma, N. Adansonia digitata leaf extract mediated synthesis of silver nanoparticles; characterization and antimicrobial studies (2015).

[33] Matsumura, Y., Yoshikata, K., Kunisaki, S. and Tsuchido, T. (2003). Mode of bactericidal action of silver zeolite and its comparison with that of silver nitrate. Applied and environmental microbiology, 69 (7), 4278-4281. 